\title{
High antithrombin III levels attenuate hemostatic activation and leukocyte activation during cardiopulmonary bypass
}

\author{
Andreas Koster, MD, a Derek Chew, MD, ${ }^{\mathrm{b}}$ Wolfgang Kuebler, MD, ${ }^{\mathrm{c}}$ Helmut Habazettl, MD, ${ }^{\mathrm{c}}$ Roland Hetzer, MD, ${ }^{\mathrm{d}}$ and \\ Herman Kuppe, MD, ${ }^{a}$ Berlin, Germany, and Adelaide, Australia
}

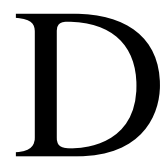
espite heparinization, cardiac surgery involving cardiopulmonary bypass $(\mathrm{CPB})$ induces a powerful activation of the coagulation and inflammation system. This is caused by the contact of the blood with the large non-endothelial surfaces of $\mathrm{CPB}$, the release and reinfusion of tissue factor-enriched blood from the operation field, the surgical trauma, and the reperfusion of ischemic tissue. As a result, the plasma coagulation factors and platelets are progressively consumed, the complement system and leukocytes are activated, and the proteases and cytokines are released. Clinical consequences of this sequel are hemorrhage, transfusions, and organ damage. Particularly in the myocardium, this systemic activation of the inflammatory system may further trigger regional reperfusion injury and thereby contribute to myocardial stunning, impaired contractility, and low cardiac output.

Thrombin plays a pivotal role in the modulation of leukocyte activation: It increases neutrophil recruitment, neutrophil rolling by the expression of endothelial P- and E-selectin, neutrophil adhesion by the expression of intracellular adhesion molecule 1 , and the production of endothelial platelet-activating factor. ${ }^{1}$

The current investigation assesses the impact of enhanced antithrombin (AT) activity, induced by a high dose of ATIII before the initiation of $\mathrm{CPB}$, on key biochemical markers of hemostatic and leukocyte activation.

\section{Methods}

Patients and anticoagulation protocol. After approval by the local ethics committee and informed consent were obtained, 80 patients undergoing elective coronary artery bypass grafting were enrolled in this prospective, randomized, controlled investigation. No patient received warfarin (Coumadin, Bristol-Myers Squibb,

From the Department of Anesthesia, Deutsches Herzzentrum Berlin, ${ }^{\text {a B Ber- }}$ lin, Germany, Department of Cardiology, Flinders Medical Centre, ${ }^{\mathrm{b}}$ Adelaide, Australia, Institute of Physiology, Freie Universität Berlin, ${ }^{\mathrm{c}}$ Berlin, Germany, and Department of Cardiothoracic and Vascular Surgery, Deutsches Herzzentrum Berlin, ${ }^{\mathrm{d}}$ Berlin, Germany.

Received for publication Nov 12, 2002; accepted for publication Jan 10, 2003.

Work attribution: Department of Anesthesia, Deutsches Herzzentrum Berlin, Germany

Supported by a grant from Grifols Germany.

Address for reprints: Andreas Koster, MD, USA, Deutsches Herzzentrum Berlin, Augustenburgerplatz 1, D-13353 Berlin (E-mail: koster@dhzb.de).

J Thorac Cardiovasc Surg 2003;126:906-7

Copyright (C) 2003 by The American Association for Thoracic Surgery

$0022-5223 / 2003 \$ 30.00+0$

doi:10.1016/S0022-5223(03)00392-1
New York, NY) or antiplatelet therapy within 10 days before surgery. Two groups with 40 patients each were formed. In group one $(\mathrm{H})$, in accordance with the departmental standard, anticoagulation was performed according to the results of the Hepcon HMS Plus (Medtronic, Minneapolis, Minn). In group two (H+ATIII), an additional bolus of $50 \mathrm{IU} / \mathrm{kg}$ ATIII (Grifols, Langen, Germany) was given with the initial bolus of heparin before CPB.

Blood samples were collected 10 minutes after the administration of the heparin bolus before CPB and after termination of CPB before protamine infusion. Anesthesia was performed using a total intravenous technique with propofol, sufentanil, midazolam, and pancuronium bromide. Normothermic CPB was accomplished with nonheparin-coated systems, membrane oxygenators, and roller pumps. None of the patients received antifibrinolytic agents or the kallikrein inhibitor aprotinin.

Laboratory analysis. Heparin anti-Xa activity was determined using the STA-Rotachrom Heparin (Roche, Mannheim, Germany). ATIII was determined using the Coamatic LR Antithrombin Chromogenix assay (Hemochron Diagnostica, Essen, Germany). Prothrombin 1 and 2 fragments (PTF1+2) were assessed using the Enzygnost F1-2 micro assay (Dade Behring, Marburg, Germany). Soluble fibrin was measured using the COATEST Soluble Fibrin assay (Chromogenix, Milano, Italy). Interleukin (IL)-6 was measured using the IL-6 enzyme-linked immunosorbent assay (DPC Biermann, Nauheim, Germany). Polymorphonuclear leukocyte (PMNL) elastase was determined with the use of a luminescence immunoassay (Auto-CliniLumat LB 952, Bertold, Wildbad, Germany).

Statistical analysis. Statistical analysis was performed with the Student $t$ test.

\section{Results}

There were no differences with regard to the biometric data, duration of $\mathrm{CPB}$, and pre-CPB data between the groups except a significantly increased level of ATIII in the H+ATIII group (Table 1).

According to the values obtained after CPB, there was no difference in anti-Xa activity in the two groups, signaling comparable heparin concentrations. There was a significantly increased ATIII concentration in the H+ATIII group, whereas the values for PTF1 +2 and soluble fibrin as markers of hemostatic activation and IL-6 and PMNL elastase as markers of leukocyte activation were significantly reduced in this group (Table 2).

No blood products were transfused before or during CPB. In the $\mathrm{H}$ group, a total of 14 red blood cell concentrates were transfused, whereas 12 were transfused in the H+ATIII group $(P=.91)$. No platelet concentrates or fresh frozen plasma was transfused in either group. The 12-hour postoperative blood loss 
TABLE 1. Biometric data, duration of CPB, and pre-CPB data

\begin{tabular}{|c|c|c|c|c|c|c|c|c|c|}
\hline & Sex & Age (yrs) & CPB (min) & ATIII (\%) & $\begin{array}{l}\text { Anti-Xa } \\
\text { (IU/mL) }\end{array}$ & $\begin{array}{l}\text { PTF1+2 } \\
\text { (nmol/L) }\end{array}$ & $\begin{array}{c}\text { SF } \\
(\mathbf{n g} / \mathrm{mL})\end{array}$ & $\begin{array}{c}\text { IL-6 } \\
\text { (ng/mL) }\end{array}$ & $\begin{array}{l}\text { PMNL elastase } \\
\text { (ng/mL) }\end{array}$ \\
\hline $\begin{array}{l}\mathrm{H} \\
\mathrm{H}+\mathrm{AT} I I I\end{array}$ & $\begin{array}{l}18 \mathrm{~F} 22 \mathrm{M} \\
19 \mathrm{~F} 21 \mathrm{M}\end{array}$ & $\begin{array}{l}65.1 \pm 12.1 \\
62.2 \pm 14.1\end{array}$ & $\begin{array}{r}98.5 \pm 27.8 \\
101.4 \pm 23.7\end{array}$ & $\begin{array}{c}76.6 \pm 12.4 \\
143.6 \pm 21.5^{*}\end{array}$ & $\begin{array}{l}4.1 \pm 1.2 \\
4.3 \pm 1.3\end{array}$ & $\begin{array}{l}0.9 \pm 0.1 \\
0.9 \pm 0.1\end{array}$ & $\begin{array}{l}11 \pm 4.1 \\
11 \pm 2.9\end{array}$ & $\begin{array}{l}16.7 \pm 8.1 \\
19.5 \pm 7.9\end{array}$ & $\begin{array}{l}69.5 \pm 21.4 \\
75.1 \pm 14.3\end{array}$ \\
\hline
\end{tabular}

${ }^{*} P<.05$.

$C P B$, Cardiopulmonary bypass; ATIII, antithrombin III; anti-Xa, activity against factor Xa; $P T F 1+2$, prothrombin fragments $1+2 ; S F$, soluble fibrin; IL, interleukin; PMNL, polymorphonuclear leukocyte.

TABLE 2. Post-CPB data

\begin{tabular}{lcccccc}
\hline & ATIII (\%) & $\begin{array}{c}\text { Anti-Xa } \\
(\mathbf{I U} / \mathbf{m L})\end{array}$ & $\begin{array}{c}\text { PTF1+2 } \\
\text { (nmol/L) }\end{array}$ & SF (ng/mL) & IL-6 (ng/mL) & $\begin{array}{c}\text { PMNL elastase } \\
\text { (ng/mL) }\end{array}$ \\
\hline H & $36.6 \pm 13.4$ & $3.5 \pm 1.1$ & $2.6 \pm 1.6$ & $16 \pm 4.5$ & $198 \pm 68.1$ & $756 \pm 198$ \\
H + ATIII & $98.1 \pm 15.5$ & $3.3 \pm 1.3$ & $1.4 \pm 2.7$ & $11 \pm 2.2$ & $37.2 \pm 9.9$ & $<09 \pm 231$ \\
$P$ & $<.01$ & .89 & $<.01$ & .02 & $<.01$ & .03 \\
\hline
\end{tabular}

was $732 \pm 365 \mathrm{~mL}$ in the $\mathrm{H}$ group and $702 \pm 431 \mathrm{~mL}$ in the $\mathrm{H}+$ ATIII group $(P=.87)$.

All patients experienced an uneventful postoperative course and were discharged from hospital on schedule.

\section{Conclusion}

Our data show that during $\mathrm{CPB}$, high concentrations of ATIII significantly reduce hemostatic activation, as shown by a significant decrease of PTF1+2 and soluble fibrin and leukocyte activation, which was evidenced by a decreased elevation of the PMNLderived cytokine IL-6 and the protease elastase. Although the current investigation included only relatively short, noncomplex $\mathrm{CPB}$ procedures and was not powered to achieve differences in clinical outcome, our data may gain clinical significance.

Despotis and colleagues ${ }^{2,3}$ demonstrated an improved preservation of the hemostatic system with subsequent reduction of blood loss and a reduction in transfusion requirements after maintenance of high heparin levels during CPB. Our data elucidate that the additional maintenance of high ATIII concentrations further contributes to a reduction of hemostatic activation. Because there is convincing evidence that cerebral fibrin microembolism is a major reason for neurocognitive dysfunction after cardiac surgery with $\mathrm{CPB},{ }^{4}$ the significant reduction of fibrin generation in the ATIII group indicates a benefit of this strategy in this regard.

Accumulation of PMNL is a key mediator of ischemia-reperfusion (I/R) injury of the heart, brain, intestine, skeletal muscle, and other tissues. ${ }^{5}$ Preclinical studies in the ischemic heart demonstrated that preischemic use of ATIII was able to prevent I/R injury, and that postischemic use of ATIII was able to inhibit reperfusion injury. ${ }^{1}$ Therefore, the maintenance of high ATIII concentrations during CPB may not only reduce systemic neutrophil activation but also effectively contribute to an attenuation of regional, especially myocardial, I/R injury.

Further studies are needed to assess whether these laboratory findings clinically translate into improved patient outcome.

The maintenance of high ATIII levels during CPB resulted in a significant reduction of biochemical key markers of hemostatic activation and leukocyte activation. Further larger clinical studies should elucidate how far these laboratory findings translate clinically into a reduction of blood loss, transfusion rates, and improved preservation of organ function.

\section{References}

1. Woodman RC, Ostrovsky L, Teoh D, Payne D, Poon M, Kubes P. Antithrombin and ischemia/reperfusion. Blood Coagul Fibrinolysis. 1998;9(Suppl 2):S7-15.

2. Despotis GJ, Joist HJ, Hogue CW Jr, Alsoufiev A, Joiner-Maier D, Santoro SA, et al. More effective suppression of hemostatic system activation in patients undergoing surgery by heparin dosing based on heparin blood concentrations rather than ACT. Thromb Haemost. 1996; 76:902-8.

3. Despotis GJ, Joist JH, Hogue CW Jr, Alsoufiev A, Kater K, Goodnough LT, et al. The impact of heparin concentration and activated clotting time monitoring on blood conservation. A prospective randomized evaluation in patients undergoing cardiac operation. $J$ Thorac Cardiovasc Surg. 1996;111:493-54.

4. Raymond PD, Marsh NA. Alterations to haemostasis following cardiopulmonary bypass and the relationship of these changes to neurocognitive morbidity. Blood Coagul Fibrinolysis. 2001;12:601-18.

5. Hansen PR. Role of neutrophils in myocardial ischemia and reperfusion. Circulation. 1995;91:1872-85. 